\title{
HISTOLOGY OF CHOROID AND RETINA AFTER MULTIPLE RECENT OCCLUSIONS OF THE RETINAL ARTERIES AS REVEALED BY SERIAL CORONAL SECTIONS OF THE GLOBE*
}

\author{
BY \\ E. NEUMANN \\ From the Department of Ophthalmology, Rothschild Hadassah University Hospital, \\ and the Hebrew University-Hadassah Medical School, Jerusalem, Israel
}

THE case reported below appears to show that associated oedema of the choroid occurs as a result of retinal arterial obstruction. The proof of the involvement of the choroid was made possible in this case by the fact that there were multiple emboli affecting separate quadrants of the retina and also through a new technique of sectioning the globe. With this technique, serial coronal sections are made from the optic disc towards the ora serrata. In this way it is possible to examine in any single slide all the retinal vessels at a given distance from the disc, to compare with ease the states of the retina and choroid in the neighbourhood of the main vascular divisions, and to map the topography of a retinal lesion.

\section{Case Report}

A 34-year-old woman was admitted to hospital 7 days after childbirth with left hemiparesis and blindness of the right eye. The previous history was negative and there was no history of hypertension or other cardiovascular disease. The patient had had seven normal deliveries. The last gestation and delivery, 7 days before the present admission, were uneventful and the patient had stayed in the maternity ward for 4 days only; 2 days later she complained of pain over the right temple and dimness of vision in the right eye. Left hemiparesis developed and there was marked weakness of the left arm. She was admitted to hospital in a stuperose condition on the sixth night following delivery.

Examination.-Blood pressure 120/80, pulse regular 88, no fever. A grade 1 systolic murmur was heard over the apex of the heart. The pelvic examination was in keeping with her post-parturient history and was otherwise negative. Left hemiparesis was present. There was hypochromic anaemia with $7 \cdot 7 \mathrm{~g}$. haemoglobin.

There was anisocoria $R>L$. The right pupil did not react to light, but the consensual reflex was present. There was pallor of the right disc with a slightly blurred margin nasally. A second ophthalmoscopic report 5 days later, again made by the neurologist, gave the additional findings of a small haemorrhage near the temporal side of the disc and fragmentation of the blood flow in the retinal vessels. Unfortunately the rest of the fundus was not discussed. 
Course.-During the days following the admission to hospital the fever rose to $38.6^{\circ} \mathrm{C}$. and did not fluctuate. Antibiotic treatment was started. 5 days after admission an attempt to perform percutaneous angiography failed. Exploration revealed a thrombus, partially organized, within the common and internal carotid arteries. Thrombectomy was performed with ligature of the right common carotid artery. The patient died 12 hours later, 6 days after the onset of symptoms.

Post mortem Examination (performed by Professor Ungar).-There was a thrombosis of the right internal carotid artery in the cervical and intracranial portions extending into the right mid-cerebral artery; extension of the thrombus distal and proximal to the ligature in the right common carotid artery; congenital anomaly of the circle of Willis; hypoplasia of the left anterior cerebral artery; encephalomacia, extensive in the right hemisphere, involving the frontal and parietal lobes, part of the corpus callosum, centrum semi-ovale, and basal ganglia; recent thrombosis of dural sinuses, transverse (bilateral) and left sigmoid, extending for some distance into the left jugular vein; acute congestion of spleen; and signs of recent delivery.

The right eye and the attached optic nerve in its entire length were obtained for histological examination. They were immersed in formalin 2 to 3 hours after death. The optic nerve was divided from the globe. A central area of infarction $12 \mathrm{~mm}$. behind the disc was found in it, and this forms the subject of a separate report.

\section{Histological Examination of the Globe}

The eye was embedded in paraffin. Coronal serial sections of the globe were made, starting at the disc, in the direction of the ora serrata, and the sections were stained with haematoxylin and eosin.

Optic Disc.-In the lamina cribrosa region there were small areas of oedema at the periphery of the nerve. The blood vessels, central artery, and vein were normal at this level and up to the first bifurcation.

Retina.-The early sections revealed temporally and inferiorly to the disc a large area of oedema involving the inner layers of the retina and considerable oedema of the choroid (Fig. 1, opposite). Slightly more anterior sections revealed a second area of retinal and choroidal oedema in the upper nasal quadrant. Fig. 2, (opposite) which is from this quadrant, shows the transition from the oedematous part of the retina and choroid to normal retina and choroid.

In the oedematous area the retina was one-and-a-half to twice the thickness of the adjoining normal retina. Although oedema of the inner layers was considerable, it was absent from the outer layers of the retina including the outer molecular layer.

Within the oedematous parts there were many arterioles completely blocked by emboli (Fig. 1) and some which showed the beginning of proliferation of endothelial cells into them (Fig. 3, overleaf). Nearer the margins of the affected retina, some of the vessels were surrounded by numerous dark-staining nuclei in various stages of karyorrhexis and pycnosis (Fig. 4, overleaf). There were a few discrete haemorrhages around some of the venules.

The ganglion cells showed increasing changes passing from the margin of the affected area towards its centre. At the margin there were cystic haloes around the cells comparable to those found in the non-oedematous parts of the retina. 
FIG. 1.-Retina and choroid below and temporal to the disc. There is considerable oedema of all the inner layers of the retina. The two arterioles are completely blocked by emboli. The underlying choroid is oedematous with considerable distension of most vessels, including the chorio - capillaris. Haematoxylin and eosin. $\times 300$.
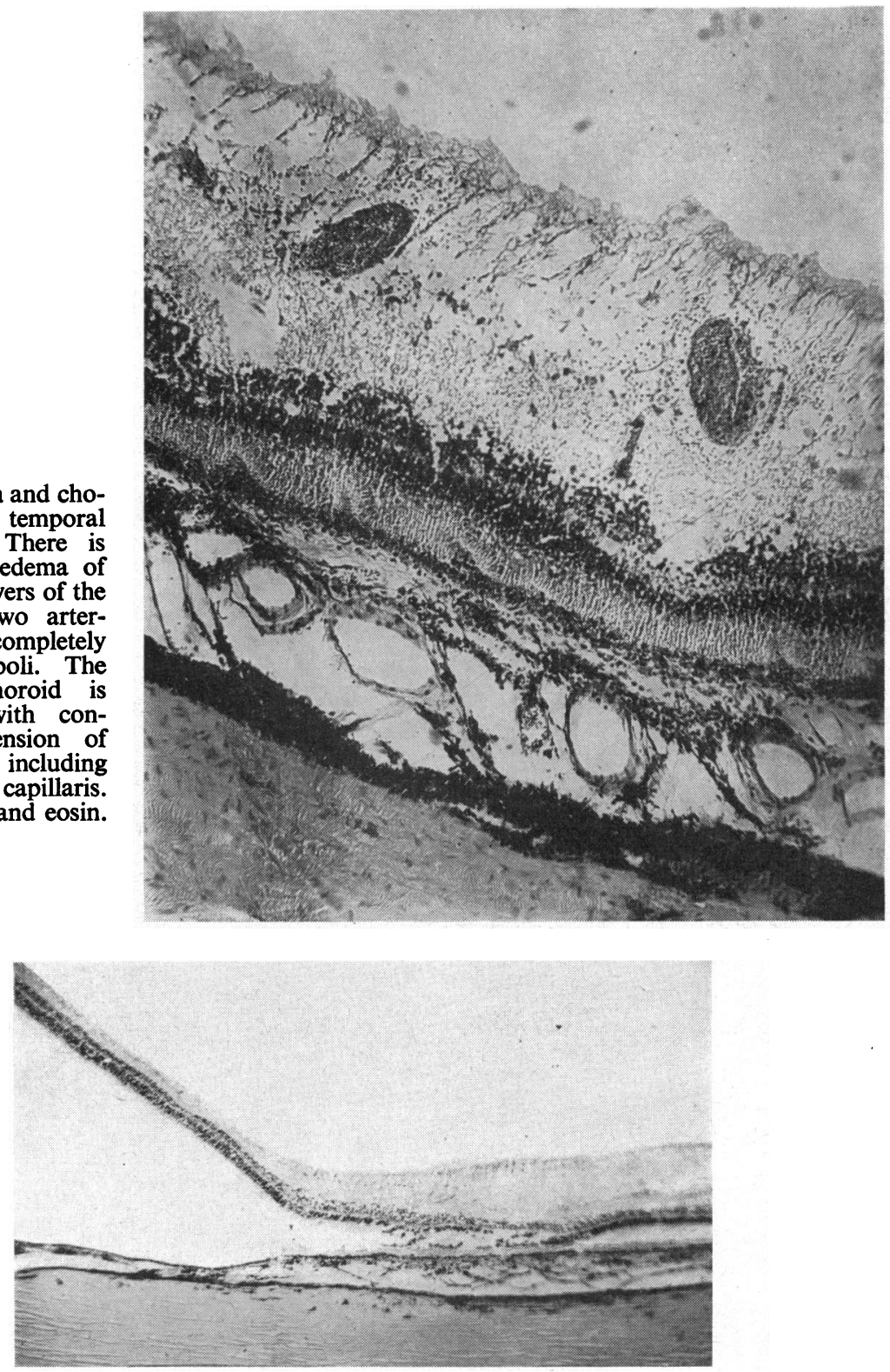

FIG. 2.-Transition from oedematous retina and choroid to normal retina and choroid. The retinal detachment is an artefact. This section is more anterior than the one shown in Fig. 1 and is from the upper nasal quadrant. The thickness of the normal retina and choroid may be compared with that of the oedematous retina and choroid. Haematoxylin and eosin. $\times 31$. 


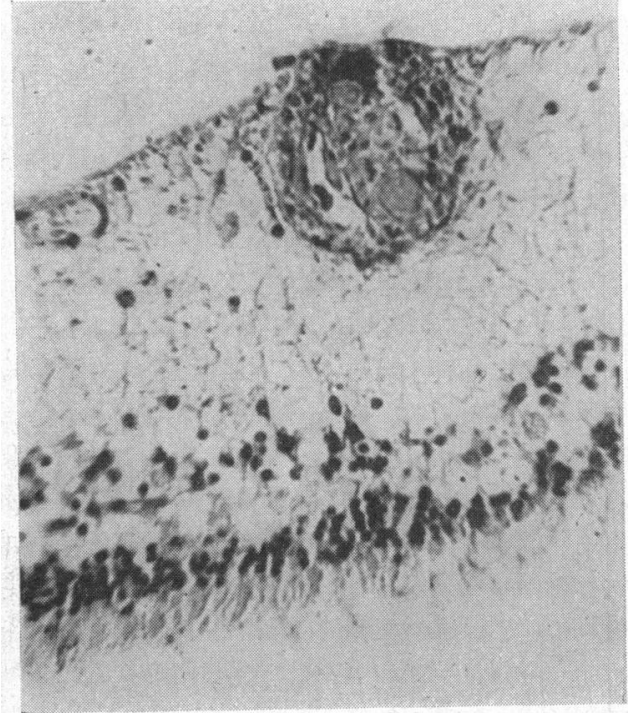

Fig. 3.-Blocked arteriole within the oedematous part of the retina with beginning proliferation of endothelial cells into embolus. Haematoxylin and eosin. $\times 335$.

Fig. 4.-Accumulation of leucocytes around a retinal blood vessel in various stages of karyorrhexis and pycnosis. Haematoxylin and eosin. $\times 563$.

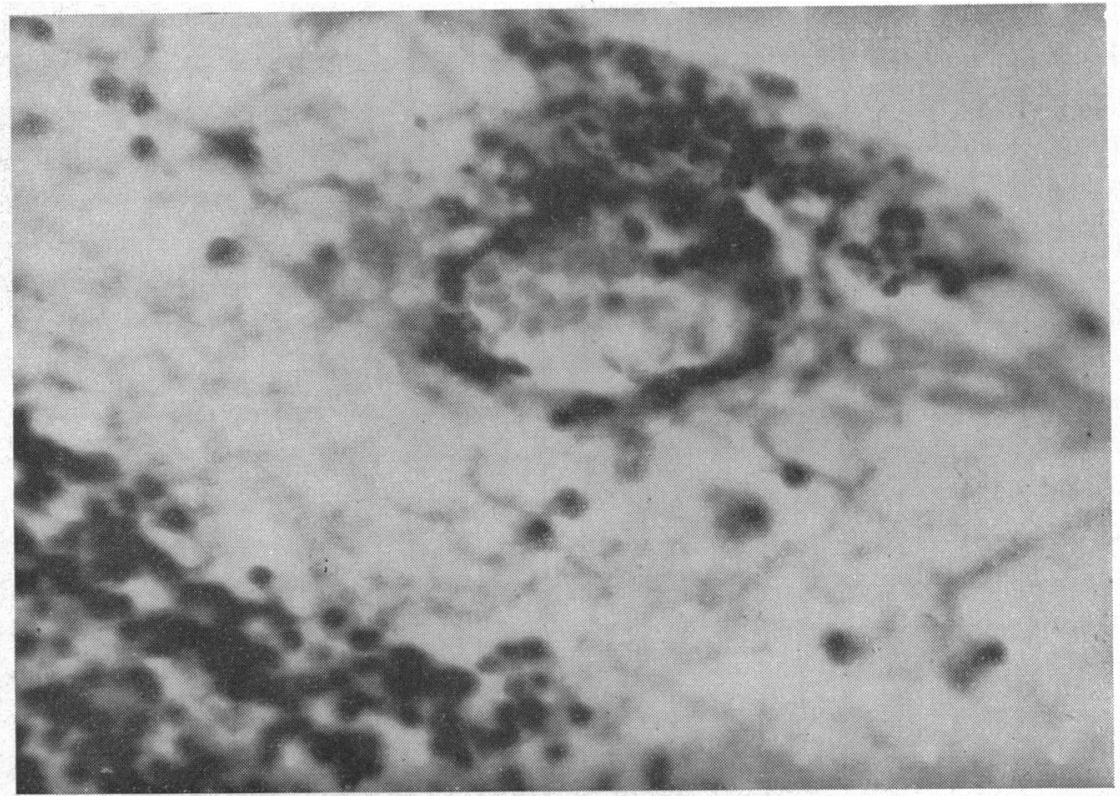

These changes were such as are usually seen post mortem. Within the affected area, swelling of the ganglion cells caused these haloes to disappear. Still closer to the centre of the affected area there was chromatolysis and disappearance of the nuclei and in places complete disappearance of the cells and of the nerve fibres (Fig. 5, opposite). 


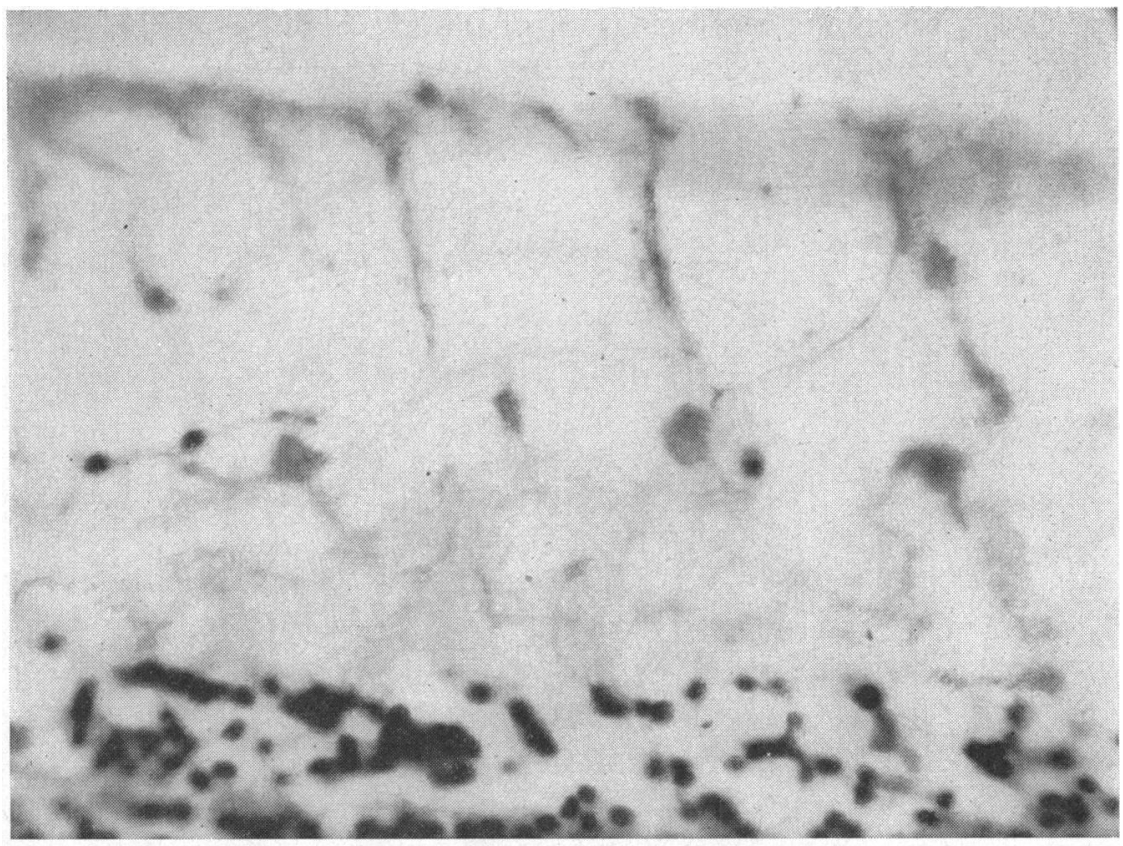

Fig. 5.-Large cystic spaces bound by Müller's fibres in retina where oedema is extreme. Chromatolysis and disappearance of nuclei of the ganglion cells. Haematoxylin and eosin. $\times 563$.

Choroid.-As already stated, immediately beneath the segments of the oedematous retina, the choroid showed considerable oedema of all its layers with distension of most vessels, including the chorio-capillaris (Figs 2 and 6). The width of the choroid in these segments was two to four times greater than that of the choroid underlying the unaffected parts of the retina. In a number of places in the oedematous choroid there was perivascular round cell infiltration.

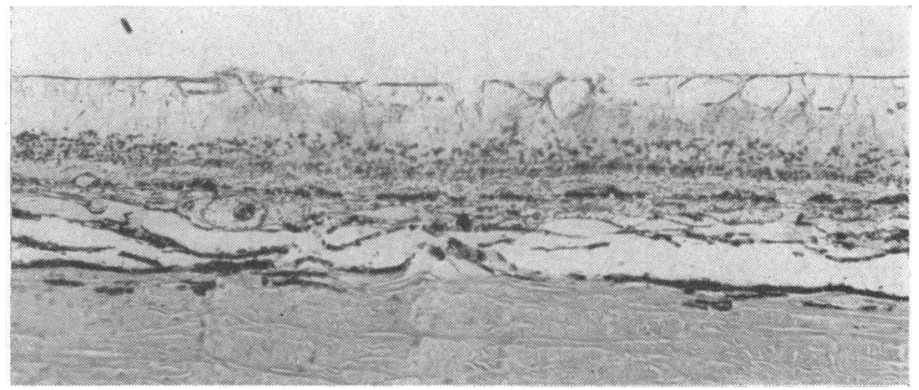

FIG. 6.-Distended chorio-capillaris under oedematous retina. Haematoxylin and eosin. $\times 90$. 
Fig. 7 is a schematic representation of the above findings, gathered from sections at seven different levels from the disc to near the ora serrata.

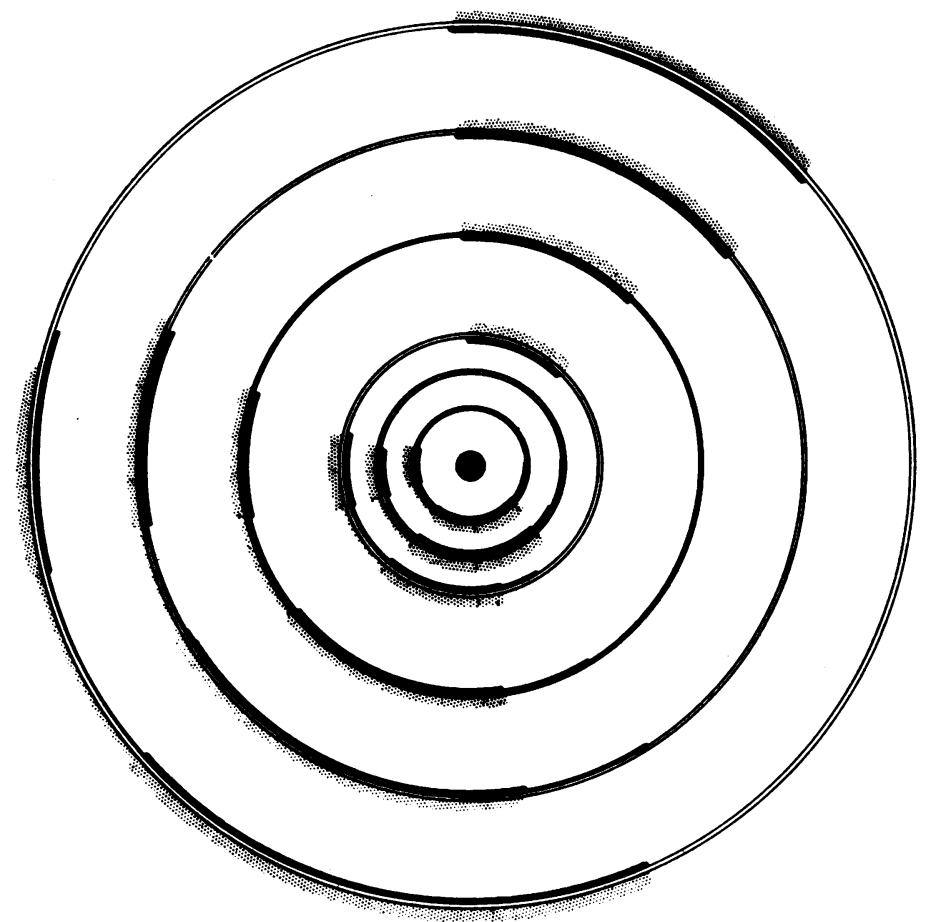

Fig. 7.-Schematic representation of sections of retina and choroid of the right eye at various distances from the disc. The outer line of each pair of circles represents the choroid, while the inner line represents the retina. Oedematous changes in the retina (dark shading) with the underlying similar changes in the choroid (dotted). These occupy the lower temporal and upper nasal quadrants.

Fig. 8 (opposite) is a schematic representation of the levels of the coronal sections of the eye which are shown in Fig. 7. The alternating oedematous and normal areas of the retina are seen together with the underlying parallel alternations in the choroid.

It will be noted in Fig. 7 that, within the larger temporal segment of oedema, there is a smaller segment towards 8 o'clock where both retinal and choroidal oedema are less marked. This figure well illustrates the advantages of coronal sectioning technique in mapping the triangular extent of such a lesion.

\section{Discussion}

Although the ophthalmic artery was found to be thrombosed, it is obvious that some circulation was maintained in both choroid and retina, as can be seen from the unaffected portions of these structures in the histological 


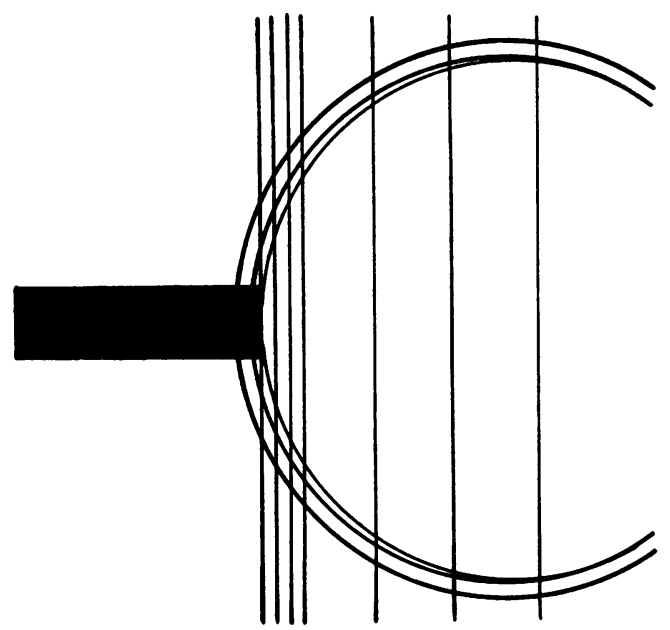

FIG. 8.-Schematic representation of levels of coronal sections of the eye shown in Fig. 7.

sections. This circulation may have come through an ophthalmic artery only partially occluded or through a collateral from the angular artery. The occlusion of several ciliary arteries may have taken place in addition to the occlusion of the retinal branches. A case of occlusion of the central retinal artery associated with embolism of the ciliary arteries was reported by Goldstein and Wexler (1933). Such ciliary occlusion would account for choroidal oedema. Yet, in the present case, it is difficult to conceive that the coextensive oedematous segments of retina and choroid, as shown in Fig. 7, should be produced by separate choroidal and retinal emboli. It would require an extreme coincidence for ciliary emboli to affect portions of the choroid overlying two separate segments of retina only. Also, occlusion of the ciliary vessels would not produce segmentary choroidal lesions parallel with retinal vascular segments. A more likely explanation of the present findings is that the choroidal engorgement and oedema is somehow related to the infarcted segments of the retina, being part of the process of retinal occlusion.

To the best of our knowledge the histological findings of retinal occlusion of no more than 6 days' duration, as in the present case, have not previously been described. No doubt the rarity of early specimens for histological examination has made the recognition of choroidal oedema difficult. However choroidal engorgement and cell infiltration have been recorded in cases of retinal obstruction by Früchte (1908), Meinshausen (1920), Mohr and Böhm (1921), and more recently by Dickson, Pritchard, Savin, and Sorsby (1944).

Early engorgement and oedema around areas of infarction are the rule rather than the exception in general pathology. The choroidal engorgement and oedema in the present case may well be such a reaction to the underlying 
ischaemic retina. Simple extension of the retinal oedema into the choroid is unlikely, since it would not account for the vascular engorgement, and moreover the outer molecular layer of the retina was free from oedema. Perhaps cytotoxic catabolic products liberated from the ischaemic retina could reach the choroid and cause both oedema and engorgement. Be the mechanism what it may, one may look on the engorgement of the choroidal vessels as a compensatory reaction between them and the retinal vessels, as suggested by Michaelson (1950). The nutritional range of the choriocapillaris into the retina is about $130 \mu$ and one may consider the full dilation of the vessels observed in the above case as an attempt to extend this range.

The whiteness of the fundus that is so characteristic of cases of retinal obstruction is possibly due in part to the underlying oedema of the choroid.

\section{Summary}

(1) A histological account is given of the early changes following an obstruction of the retinal vessels of no more than 6 days' duration.

(2) Oedema of the choroid appears to be an integral part of the process and its mechanism and function are discussed.

(3) Serial coronal sectioning of the globe facilitated the above findings.

(4) The possible role of choroidal oedema in contributing to the fundal - appearance is suggested.

I wish to express my gratitude to Prof. I. C. Michaelson for his advice, to Prof. H. Ungar for the report of the general post mortem findings, and to Mr. M. Ivry for his help with the illustrations.

\section{REFERENCES}

Dickson, W. E. C., Pritchard, G. C., Savin, L. H. and Sorsby, A. (1944). Brit. J. Ophthal., 28, 1 .

FRÜCHTE, W. (1908). Klin. Mbl. Augenheilk., 46 (n.s. 5), 245.

GOLDSTEIN, I., and WEXLER, D. (1933). Arch. Ophthal. (Chicago), 10, 70.

MeInshausen, W. (1920). Klin. Mbl. Augenheilk., 65, 199.

Michaelson, I. C. (1950). "XVI Concilium Ophthalmologicum. 1950. Britannia Acta", vol. 1, p. 605 . B.M.A., London.

MoHR, T., and BöHM, L. (1921). Klin. Mbl. Augenheilk., 66, 812. 